\title{
COHOMOLOGY IN ABSTRACT UNIT GROUPS
}

\author{
YUKIYOSI KAWADA
}

A. H. Clifford and S. MacLane [2] considered in 1941 the group of factor-sets $H^{2}(\Gamma, U)$ of a finite group $\Gamma$ over its abstract unit group $U$. They proved the main theorem to the effect that $H^{2}(\Gamma, U)$ is isomorphic to the multiplicator $M$ of $\Gamma$ defined by I. Schur and also several other theorems under the assumption that $\Gamma$ is a solvable group. They conjectured that these should hold for general finite groups $\Gamma$. In $1942 \mathrm{~A}$. Weil proved the main theorem for general finite groups $\Gamma$, but this result was not published. ${ }^{1}$ In this short note we shall prove that all the theorems in [2] are valid for general finite groups $\Gamma$, and also we shall extend their results for all (positive, zero, and negative) dimensional cohomology groups. ${ }^{2}$

1. We shall first prove a general lemma on cohomology groups. Let $\Delta$ be a finite group, and let $E$ be a $\Delta$-module. Suppose that $A_{1}, A_{2}$ are two $\Delta$-submodules which are disjoint: $A_{1} \cap A_{2}=0$. Then we have the following commutative diagram such that each row and each column are exact:

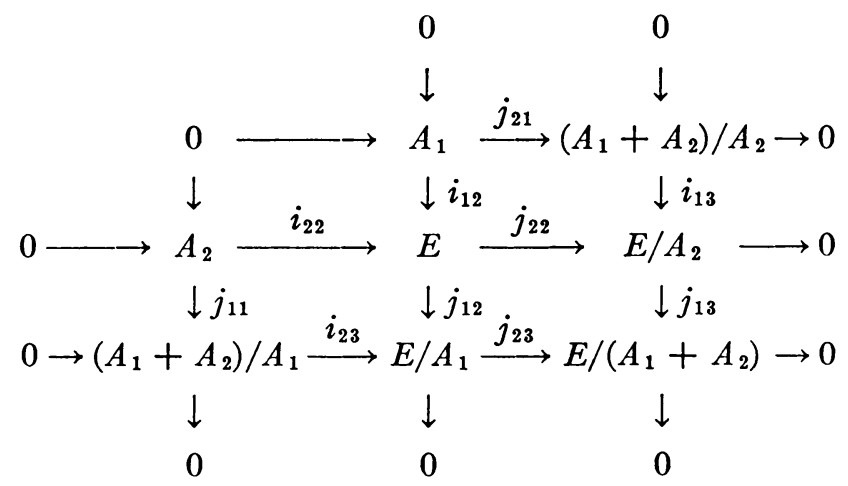

Let us denote, in general, by $H^{r}(\Delta, A)$ the $r$-cohomology group of a group $\Delta$ over a $\Delta$-module $A$.

Lemma. A ssume that $H^{r}(\Delta, E)=0$ for $r=0, \pm 1, \pm 2, \cdots$. Then we have for all $r=0, \pm 1, \pm 2, \cdots$,

Received by the editors April 1, 1954.

1 The author wishes to express his thanks to Professor Saunders MacLane who kindly told him this fact and allowed him to read an unpublished manuscript about it.

2 For the definition of negative dimensional cohomology groups of a finite group and for the properties of cohomology groups see, for example, Artin-Tate [1]. 
$(\mathrm{I})^{*} \quad H^{r}\left(\Delta, E / A_{1}\right) \stackrel{\delta}{\cong} B^{r+1}\left(\Delta, A_{1}\right) \stackrel{j^{*}}{\cong} H^{r+1}\left(\Delta,\left(A_{1}+A_{2}\right) / A_{2}\right)$,

(II)* $0 \rightarrow H^{r}\left(\Delta, E / A_{2}\right) \stackrel{j^{*}}{\rightarrow} H^{r}\left(\Delta, E /\left(A_{1}+A_{2}\right)\right)$

$$
\stackrel{\delta}{\rightarrow} H^{r+1}\left(\Delta,\left(A_{1}+A_{2}\right) / A_{2}\right) \stackrel{i^{*}}{\rightarrow} 0(\text { exact })
$$

and similar formulas hold by interchanging the subscripts 1 and 2,

(III)* $H^{r}\left(\Delta, E /\left(A_{1}+A_{2}\right)\right)=j_{23}^{*}\left(H^{r}\left(\Delta, E / A_{1}\right)\right)+j_{13}^{*}\left(H^{r}\left(\Delta, E / A_{2}\right)\right)$.

Proof. (i) (I)* is evident by our assumption $H^{r}(\Delta, E)=0$. (ii) Since $\left(i_{13}\right)^{*}=\left(j_{22}\right)^{*} \circ\left(i_{12}\right)^{*} \circ\left(j_{21}^{-1}\right)^{*}$ and $\left(i_{12}\right)^{*}=0$ by our assumption, we have $\left(i_{13}\right)^{*}=0$. Hence we get (II)* by the exact sequence of cohomology groups derived from the 3 rd column of the diagram (1). (iii) From (1) follows

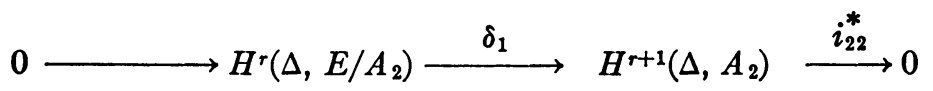

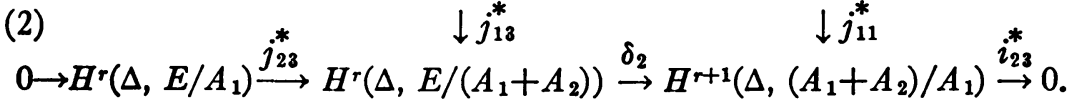

Here $j_{13}^{*}$ is an into-isomorphism and $\delta_{1}, j_{11}^{*}$ are onto-isomorphisms. Hence $j_{13}^{*}\left(H^{r}\left(\Delta, E / A_{2}\right)\right)$ is a splitting system of representatives of $H^{r}\left(\Delta, E /\left(A_{1}+A_{2}\right)\right) \bmod j_{23}^{*}\left(H^{r}\left(\Delta, E / A_{1}\right)\right)$. This proves (III)*, q.e.d.

2. Let $\Gamma$ be a finite group of order $n$, and $\Gamma(Z)$ be its group ring over the integers $Z$. Put $u=\sum_{\sigma \in \Gamma} \sigma \in \Gamma(Z)$. Then by definition the factor group $U=\Gamma(Z) / Z u$ is the abstract unit group of $\Gamma$. Now let $\Delta$ be an arbitrary subgroup of $\Gamma$. Let us take $E=\Gamma(Z), A_{1}$ $=\sum_{\sigma \neq 1} Z(1-\sigma)$, and $A_{2}=Z u$. Clearly $A_{1} \cap A_{2}=0$. Since $E=\Gamma(Z)$ is $\Delta$-free, the assumption in the lemma is satisfied. Hence we can apply the lemma. Here we may identify $E / A_{1}=Z$ and $j_{12}=\operatorname{tr}$, where $\operatorname{tr}\left(\sum_{\sigma} a_{\sigma} \cdot \sigma\right)=\sum_{\sigma} a_{\sigma} \in Z\left(a_{\sigma} \in Z\right)$. Then the 3rd row of the diagram (1) may be replaced by

$$
0 \rightarrow n Z \stackrel{i_{23}}{\rightarrow} Z \stackrel{j_{23}}{\rightarrow} Z / n Z \rightarrow 0
$$

where $\Delta$ operates on these modules trivially. Also $j_{13}$ and $j_{11}$ become the homomorphism tr induced in $U(\rightarrow Z / n Z)$ and $Z u(\rightarrow n Z)$ respectively. Finally put $U_{0}=\left(A_{1}+A_{2}\right) / A_{2}$, which is the kernel of the mapping $\operatorname{tr} U \rightarrow Z / n Z$. By these substitutions we have the following formulas from our lemma:

For all $r=0, \pm, \pm 2, \cdots$ 
(I) ${ }_{1} \quad H^{r}(\Delta, U) \stackrel{\delta}{\cong} H^{r+1}(\Delta, Z)$,

(I) ${ }_{2} \quad H^{r-1}(\Delta, Z) \stackrel{j_{21}^{*} \cdot \delta}{\cong} H^{r}\left(\Delta, U_{0}\right)$,

(II) $)_{1} \quad 0 \rightarrow H^{r}(\Delta, U) \stackrel{\operatorname{tr}^{*}}{\rightarrow} H^{r}(\Delta, Z / n Z) \stackrel{\delta}{\rightarrow} H^{r+1}\left(\Delta, U_{0}\right) \stackrel{i^{*}}{\rightarrow} 0$ (exact),

(II) $)_{2} \quad 0 \rightarrow H^{r}(\Delta, Z) \stackrel{i^{*}}{\rightarrow} H^{r}(\Delta, Z / n Z) \stackrel{\delta}{\rightarrow} H^{r+1}(\Delta, n Z) \stackrel{i^{*}}{\rightarrow} 0($ exact $)$,

(III) $H^{r}(\Delta, Z / n Z)=j_{23}^{*}\left(H^{r}(\Delta, Z)\right)+\operatorname{tr}^{*}\left(H^{r}(\Delta, U)\right)$,

where $\Delta$ operates trivially on $Z, n Z$ and $Z / n Z$.

Now we get several theorems in [2] as corollaries of these formulas. Namely, from $(\mathrm{I})_{2}$ follows

(i) $H^{0}\left(\Delta, U_{0}\right) \cong H^{-1}(\Delta, Z)=0 ; H^{1}\left(\Delta, U_{0}\right) \cong H^{0}(\Delta, Z) \cong Z / m Z$ where $m$ is the order of $\Delta ; H^{2}\left(\Delta, U_{0}\right) \cong H^{1}(\Delta, Z)=0$ (formulas (1), (2) in $\S 6$ and corollary in $\$ 1$ of $[2])$. From (II) $)_{1}$ follows

(ii) $\operatorname{tr}^{*}: H^{2}(\Delta, U) \rightarrow H^{2}(\Delta, Z / n Z)$ is an into-isomorphism (Theorem 1.A of $[2])$,

(iii) $i^{*}\left(H^{1}\left(\Delta, U_{0}\right)\right)=0$ in $H^{1}(\Delta, U)$ (Theorem 1.B of [2]).

From (II) $)_{1}$ and $H^{2}\left(\Delta, U_{0}\right)=0$ follows

(iv) $\operatorname{tr}^{*}: H^{1}(\Delta, U) \rightarrow H^{1}(\Delta, Z / n Z)$ is an onto-isomorphism (Theorem 2.B of $[2])$.

3. Let $\Omega$ be an algebraically closed field of characteristic not dividing the order $n$ of $\Gamma$. Then the multiplicator $M$ of $\Gamma$ is defined by I. Schur as $M=H^{2}\left(\Gamma, \Omega^{*}\right)$, where $\Gamma$ acts trivially on the multiplicative group $\Omega^{*}$. Let $W$ be the group of all the roots of unity in $\Omega$. Consider the exact sequence $1 \rightarrow W \rightarrow \Omega^{*} \rightarrow \Omega^{*} / W \rightarrow 1$. Since the group $\Omega^{*} / W$ is uniquely divisible, so $H^{r}\left(\Delta, \Omega^{*} / W\right)=0$ for all $r$. Hence we have $M=H^{2}\left(\Gamma, \Omega^{*}\right) \cong H^{2}(\Gamma, W) \cong H^{2}(\Delta, Q / Z)$, where $Q$ is the additive group of rationals. Let the homomorphism aver. (=average) be defined on $\Gamma(Z)$ by

$$
\text { aver. }\left(\sum_{\sigma} a_{\sigma} \cdot \sigma\right)=\frac{1}{n} \sum_{\sigma} a_{\sigma}=\frac{1}{n} \operatorname{tr}\left(\sum_{\sigma} a_{\sigma} \cdot \sigma\right) \in Q .
$$

This homomorphism aver. induces also the homomorphism aver.: $U \rightarrow Q / Z$. A. Weil proved the main theorem in [2] for general $\Gamma$ in the form:

(v) aver. ${ }^{*}: H^{2}(\Gamma, U) \rightarrow H^{2}(\Gamma, Q / Z)$ is an onto-isomorphism. For the sake of completeness we shall give here a proof which is essentially the same as that of $\mathrm{A}$. Weil. Let us consider the commutative diagram: 


$$
\begin{aligned}
0 \rightarrow Z u \rightarrow \Gamma(Z) \rightarrow & U \rightarrow 0 \\
& \downarrow \phi_{1} \quad \downarrow \phi_{2} \quad \downarrow \phi_{3} \\
0 \rightarrow & \rightarrow \quad Q \rightarrow Q / Z \rightarrow 0
\end{aligned}
$$

where $\phi=$ aver. Since $H^{r}(\Delta, Q)=H^{r}(\Delta, \Gamma(Z))=0$ for all $r$, this diagram induces the commutative diagram:

$$
\begin{array}{cc}
0 \rightarrow H^{r}(\Delta, U) & \stackrel{\delta_{1}}{\rightarrow} H^{r+1}(\Delta, Z u) \rightarrow 0 \\
\downarrow \phi_{3}^{*} & \downarrow \phi_{1}^{*} \\
0 \rightarrow H^{r}(\Delta, Q / Z) \stackrel{\delta_{2}}{\rightarrow} H^{r+1}(\Delta, Z) \rightarrow 0 .
\end{array}
$$

Here $\phi_{1}^{*}$ is an onto-isomorphism, so is $\phi_{3}^{*}=\delta_{2}^{-1} \circ \phi_{1}^{*} \circ \delta_{1}$. Hence we get

(IV) aver. ${ }^{*}: H^{r}(\Delta, U) \rightarrow H^{r}(\Delta, Q / Z)$ is an onto-isomorphism for all $r$. The relation between $\operatorname{tr}^{*}$ and aver. ${ }^{*}$ on $H^{r}(\Delta, U)$ is given as follows. Let $\psi$ be the homomorphism defined by $\psi(\alpha)=\alpha \times(1 / n)$ on $Z(\rightarrow Q)$, $n Z(\rightarrow Z)$ and $Z / n Z(\rightarrow Q / Z)$ respectively. Then we have aver. $=\psi \circ \mathrm{tr}$, and $\psi$ induces the homomorphism: $H^{r}(\Delta, Z / n Z)^{\psi *} \rightarrow H^{r}(\Delta, Q / Z)$. Then we have

(V) $\psi^{*} \circ j_{23}^{*}\left(H^{r}(\Delta, Z)\right)=0$ and $\psi^{*}$ is an isomorphism of $\operatorname{tr}^{*}\left(H^{r}(\Delta, U)\right)$ onto $H^{r}(\Delta, Q / Z)$.

Proof. Let us consider the commutative diagram:

$$
\begin{aligned}
0 \rightarrow & n Z \stackrel{i_{1}}{\rightarrow} Z \stackrel{i_{1}}{\rightarrow} Z / n Z \rightarrow 0 \\
& \downarrow \psi_{1_{1}} \downarrow \psi_{2} \quad \downarrow \psi_{3} \\
0 \rightarrow & \stackrel{\stackrel{i_{2}}{\rightarrow}}{\rightarrow} \stackrel{\stackrel{i_{2}}{\rightarrow}}{\rightarrow} Q / Z \rightarrow 0 .
\end{aligned}
$$

Since $\left(\psi_{2}\right)^{*}=0$ by $H^{r}(\Delta, Q)=0$, we have $\psi_{3}^{*} \circ j_{1}^{*}=j_{2}^{*} \circ \psi_{2}^{*}=0$. Since $\phi_{3}^{*}=\psi_{3}^{*} \circ j_{13}^{*}$ and $\phi_{3}^{*}\left(j_{13}^{*}\right)$ is an isomorphism-onto (-into), so $\psi_{3}^{*}$ is an onto-isomorphism, q.e.d. These considerations actually cover Theorem 2.A of [2].

\section{REFERENCES}

1. E. Artin and J. Tate, Algebraic functions and algebraic numbers, Lecture notes at Princeton University, vol. 2, to be published.

2. A. H. Clifford and S. MacLane, Factor-sets of a group in its abstract unit group, Trans. Amer. Math. Soc. vol. 50 (1941) pp. 385-406.

The Institute for Advanced Study and

$$
\text { UNIVERSITY OF TOKYO }
$$

